\title{
La radio pública estatal en España como medio de empoderamiento de las personas a través de la alfabetización científica: A hombros de gigantes y Entre probetas
}

\author{
Public radio in Spain with national dissemination as a means \\ of empowering people through scientific literacy: \\ A hombros de gigantes and Entre probetas
}

\author{
Autora \\ Dra. Ana Bellón Rodríguez \\ http://orcid.org/0000-0002-8779-1110 \\ Dra en Periodismo por la Universidad de Santiago de Compostela, \\ Responsable de Comunicación del Consejo Superior de Investigaciones Científicas en Galicia \\ Profesora asociada en la Facultad de Ciencias de la Comunicación de la USC
}

\section{RESUMEN}

En la última década la sociedad española ha incrementado su interés por la I+D+i, y la radio se ha situado como el tercer medio preferido para informarse sobre temas científicos. Sin embargo, el nivel de educación científica de la población española continúa siendo bajo y la sociedad percibe que los medios prestan a la ciencia una atención insuficiente. En este contexto, se realiza un estudio exploratorio-descriptivo con una metodología mixta focalizado en la radio pública estatal en España para saber si funciona como canal de alfabetización científica y si cumple su cometido de servicio público. Se busca en las parrillas de las seis emisoras de RTVE, se localizan 46 espacios y se seleccionan dos casos de estudio: "A hombros de gigantes" (RNE), "Entre probetas" (Radio 5). Se indaga en ellos con la entrevista en profundidad con sus promotores y el análisis de contenido de cuatro programas. Se observa que la radio pública estatal sí cumple su papel de canal de alfabetización científica en España, si bien aún queda

\section{ABSTRACT}

In the last decade the Spanish society has increased its interest in $\mathrm{I}+\mathrm{D}+\mathrm{i}$, and radio has been ranked as the third media to learn about science. However, the level of science education of the Spanish population remains low and society perceives that the media don't pay attention to the science. In this context, we did an exploratory-descriptive study with a mixed methodology. The study focused on state public radio in Spain to see if it works as a channel of scientific literacy and if it fulfills its mission of public service. It searches in the programming of the six radio stations of RTVE, 46 programmes are located and two case studies are selected: "A hombros de gigantes" (RNE) and "Entre probetas"(Radio 5). It delves into them with in-depth interviews with their promoters and content analysis of four programs. It is noted that the state public radio fulfills its role as a channel of scientific literacy in Spain, although there is still a long way to go, since both spaces are characterized by to be single-person initiatives with 
mucho por avanzar, pues los dos espacios se caracterizan por ser iniciativas unipersonales, con un fuerte peso de la polivalencia profesional y apoyados por colaboradores externos. Se emiten en las franjas de menor audiencia, apuestan por la radio a la carta y por las redes sociales, pero aún no promueven iniciativas específicas para la participación del oyente.

PALABRAS CLAVE: Alfabetización científica; España; espacios; radio de calidad; radio pública; servicio público a strong weight of multi-skilling and supported by collaborators external. They are issued on the fringes of smaller audience, betting on the radio on demand and social networks, but do not promote specific initiatives to involve the listener.

KEYWORDS: Scientific literacy; Spain; spaces; quality radio; public radio; public service

FUENTES DE FINANCIACIÓN: Este artículo forma parte de los trabajos exploratorios para el marco contextual y referencial del proyecto de investigación "Usos y preferencias informativas en el nuevo mapa de medios en España: modelos de periodismo para dispositivos móviles". (Referencia: CSO2015-64662-C4-4-R).

\section{INTRODUCCIÓN}

La ciencia, entendida como el conjunto de conocimientos obtenidos mediante la observación y el razonamiento, sistemáticamente estructurados y de los que se deducen principios y leyes generales (RAE, 2016), tiene ante sí un reto: llegar a la sociedad, ser comprendida y constituir un tema objeto de interés para el público general, para el público no especializado.

Hacerla extensible para aumentar el nivel cultural de la ciudadanía, lejos de ser un lujo, es una imperiosa necesidad que beneficia a todos (Fundación Cotec, 2006: 14). Vivimos en la denominada Sociedad del Conocimiento, lo que implica, según palabras de Mateo (2006: 145), "que el saber y el conocimiento son parámetros que gobiernan y condicionan la estructura y la composición de la sociedad actual y son, también, las mercancías e instrumentos determinantes del bienestar y progreso de los pueblos". Se debe, por tanto, garantizar su aprovechamiento compartido, al tratarse de bienes públicos que corresponde a todos (UNESCO, 2005: 17-25). En ese saber y conocimiento se incluye, naturalmente, la I+D+i, con todas las áreas: desde las conocidas popularmente como "ciencias duras" a las "ciencias blandas" (Solís, 2002).
Estamos ante un derecho, el del acceso por parte de la sociedad al saber y conocimiento científico, recogido en la Declaración Universal de los Derechos Humanos adoptada por la Asamblea General de las Naciones Unidas (1948), donde se establece que "toda persona tiene derecho a tomar parte libremente en la vida cultural de la comunidad, a gozar de las artes y a participar en el progreso científico y en los beneficios que de él resulten" (artículo 27).

En el marco legal europeo, Pérez Tornero (2009) valora la incorporación de la Directiva de Servicios Audiovisuales por parte de todos los Estados miembros de la Unión Europea como un paso clave para la alfabetización mediática, pues considera que con ello se "instauraba como un elemento esencial del sistema comunicativo europeo, al introducirse por primera vez en la regulación del sistema mediático la necesidad de promover en todos los sectores de la sociedad las habilidades, los conocimientos y las capacidades de comprensión que permitan a los ciudadanos utilizar con seguridad y eficacia los medios".

En el marco legal español cabe citar que en la Constitución (1978) se establece que "los 
poderes públicos promoverán y tutelarán el acceso a la cultura, a la que todos tienen derecho, y la ciencia y la investigación científica en beneficio del interés general" (artículo 44) y en la Ley 14/2011, de 11 de junio, de la Ciencia, la Tecnología y la Innovación que "las Administraciones Públicas fomentarán las actividades conducentes a la mejora de la cultura científica y tecnológica de la sociedad a través de la educación, la formación y la divulgación, y reconocerán adecuadamente las actividades de los agentes del sistema español de Ciencia, Tecnología e Innovación en este ámbito" (artículo 38).

Según Falk y Dierking (2010), "dado que la mayor parte de la educación científica de una persona se hace fuera de un ambiente formal, la mejor manera de aumentar la comprensión del público en relación a la ciencia está en el restante de su vida". Y es ahí donde tienen un papel determinante los medios de comunicación, agentes sobre los que Mccombs (2006) ha descrito el importante, y a menudo polémico, papel a la hora de determinar cuáles son los asuntos que están en el centro de la atención y acción públicas. Ramos (1995), por su parte, ha reflexionado sobre cómo el ser humano accede al conocimiento a través de los medios, incidiendo en que "no sólo aportan información, sino que también señalan lo que es importante y trivial mediante lo que muestran y omiten".

En este sentido, son de interés los datos aportados por la VII Encuesta de Percepción Social de la Ciencia y la Tecnología, elaborada por la Fundación Española para la Ciencia y la Tecnología (FECYT) a través de un cuestionario semiestructurado, llevado a cabo mediante entrevista personal y domiciliaria, a personas residentes en España durante cinco o más años, de 15 años de edad en adelante. Los resultados apuntan que hay un déficit informativo en temas científicos del -0,43, pues es mayor el grado de interés que manifiestan tener los encuestados frente al nivel de información del que disponen en los medios (FECYT, 2015: 317-362).
En el ranking de temas de mayor interés social, la ciencia y la tecnología ocupan el noveno lugar (15\%), si bien dicho interés ha ido en aumento desde la primera edición de la encuesta (2004), tal y como se observa en el siguiente gráfico:

\section{Gráfico 1. Evolución del interés espontáneo por la ciencia y la tecnología}

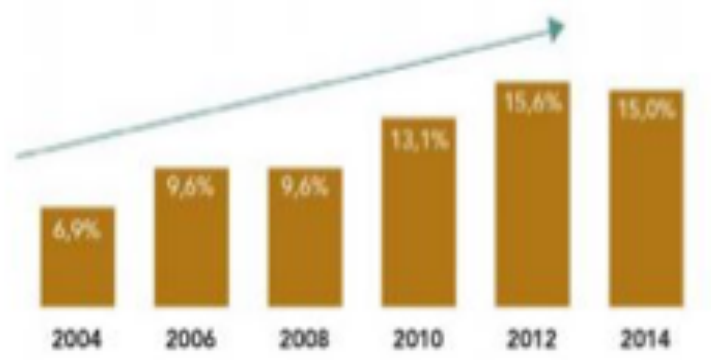

Fuente: FECYT (2015)

La relación de la ciencia con los medios de comunicación puede calificarse, cuanto menos, como compleja. Ribas (2002) ha analizado el periodismo científico desde el estudio de las prácticas profesionales, lo que permite conocer los fenómenos de distorsión que afectan a la información generada por los medios. A ello se unen tensiones entre los dos colectivos implicados: los científicos y los periodistas. Ambos no suelen coincidir en qué es noticia y discrepan en cómo hay que transmitir la información (Nelki, 1990: 147-161). Y es que difundir la ciencia de manera útil y valiosa tanto para la propia ciencia como para la sociedad sigue siendo un reto cuya solución, según la European Commission (2012) "no consiste en ofrecer más información sobre ciencia, sino en una comunicación y un diálogo más eficaces".

Para que los medios de comunicación divulguen la I+D+i es fundamental la conso- 
nancia de los siguientes factores. En primer lugar, el compromiso de la comunidad científica, a la que no siempre resulta fácil vencer la resistencia de dar a conocer su trabajo exclusivamente a través de las revistas especializadas y que debe mejorar el círculo de la comunicación y debate de su investigación (Bik y Goldsrein, 2013). En segundo lugar, la labor los Departamentos de Comunicación de los agentes de los sistemas de I+D+i, que deben garantizar un volumen de información científica homogéneo teniendo en cuenta aspectos de la profesión como la regla de las Ws o los valores noticia (Bellón, 2015: 57). En tercer lugar, la especialización de los periodistas que desarrollan su labor en los medios para satisfacer las necesidades de información de unas audiencias que disponen cada vez de un mayor dominio de los contenidos (Quesada, 1998: 14).

La especialización periodística en I+D+i es clave para que la ciencia llegue a los medios y a la sociedad, pues permite a los profesionales que manejan esa información analizar, explicar e interpretar procesos con rigurosidad, adaptándose a las necesidades del público receptor (Berganza, 2005: 60). Comienza a vislumbrarse en la década de los 70 debido a la necesidad de una interpretación adecuada de la naturaleza científica y tecnológica del progreso (Bell, 1973) y a varias crisis (Fernández del Moral, 1993). Debe dar respuesta a especialización por contenidos, por sectores de audiencias y por medios de comunicación (Quesada, 1998), logrando una visión global, asequible y coherente de todas las parcelas del conocimiento (Berganza, 2005: 57) y mejorando, a su vez, la calidad de la información periodística (Tuñón, 1993: 96).

Para que un saber sea objeto de información periodística especializada debe ser socialmente relevante (Muñoz, 1997), y no hay duda de que la ciencia lo es. Por ello, en pleno siglo XXI el periodismo científico es una de las principales áreas de especialización, al abordar la información que procede de los avances, intereses o hechos suminis- trados por la ciencia (Elías, 2008) y ejercer de nexo entre el conocimiento científico y la sociedad (Sanmartín, 2003).

En palabras de Méndez (2007), "la transmisión del conocimiento científico se hace de la misma manera en que se transmite un contenido económico, político o deportivo: adaptándolo al medio". De entre los medios de comunicación tradicionales la radio reúne una serie de características que condicionan el tratamiento de los temas científicos $y$, en algunos casos, suponen una dificultad añadida para divulgarlos sin perder el rigor (Ortiz y Pérez, 2006: 69). Elías (2008: 205) apunta que "entre sus limitaciones destaca la fugacidad del mensaje radiofónico, lo que implica que no pueda ser muy complejo, y entre sus ventajas que se trata de un medio relativamente barato, lo que implica que puede suponer un canal importante de alfabetización científica".

Desde su nacimiento, la radio no ha dejado de redefinir su papel dentro del panorama mediático, poniendo fin a la radio espectáculo y orientándola hacia el terreno de la información, la opinión y el entretenimiento (Pousa \& Yaguana, 2013: 137). Desde los inicios del siglo XXI se ha enfrentado a un panorama mediático altamente competitivo, en el que se enmarca el auge de Internet, el nacimiento de las redes sociales o nuevas formas de consumo de información a través de los dispositivos móviles. En ese contexto, ha conseguido mantenerse en niveles elevados dentro del ranking de medios. 


\section{Gráfico 2. Audiencia general de medios. Penetración (\%)}

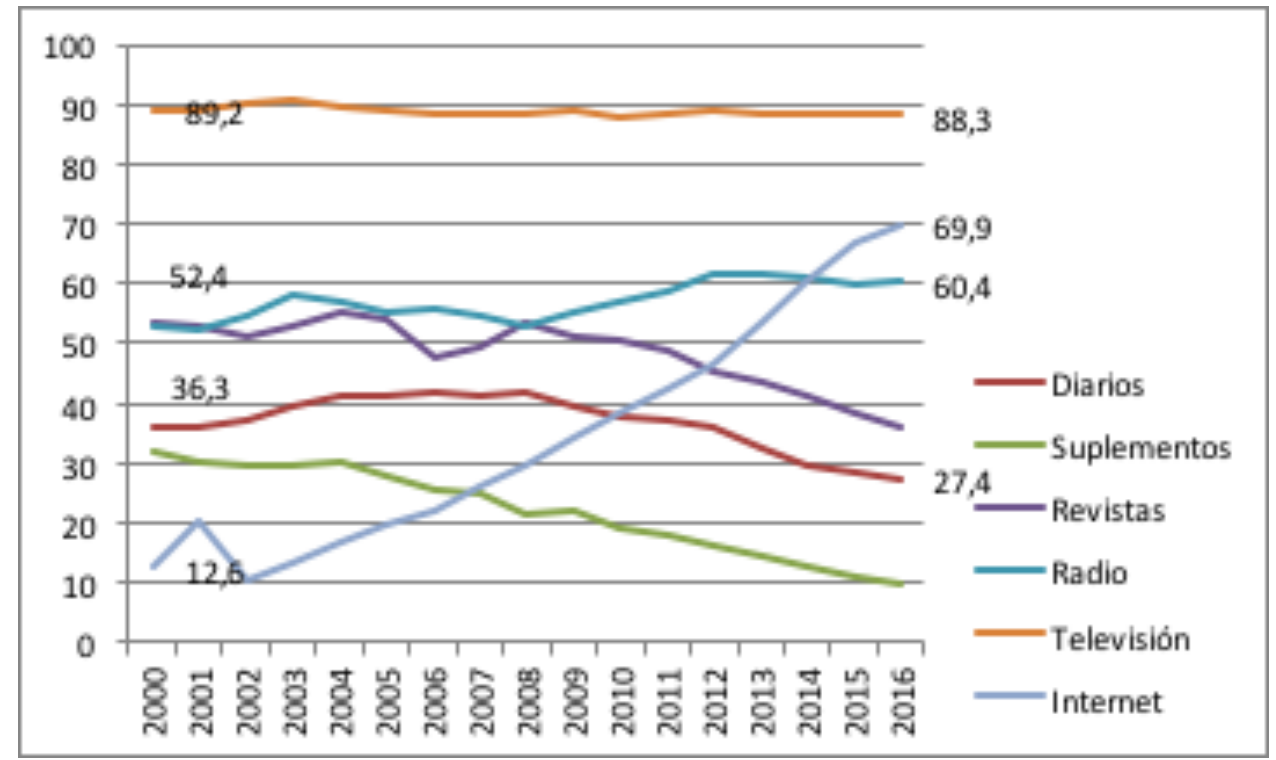

Fuente: elaboración propia a partir de datos del EGM (2000-2016)

En la última década la radio se ha convertido, además, en una fuente frecuente y de confianza para informarse sobre $I+D+i$, según se recoge en las distintas Encuestas de Percepción Social de la Ciencia y la Tecnología de la FECYT, si bien los encuestados perciben que presta poca atención a estas temáticas.
En 2004 la televisión era, con un 62,5\%, el medio al que más personas recurrían en España para informarse sobre ciencia y tecnología, seguida de la prensa $(33,1 \%)$, la radio $(31,6 \%)$ e Internet (22,4\%). En 2014 la televisión ha revalidado su posición, el segundo puesto lo ocupa ahora Internet, el tercero la radio y el cuarto la prensa.

\section{Gráfico 3. Principales medios para informarse sobre ciencia y tecnología en 2014 (\%)}

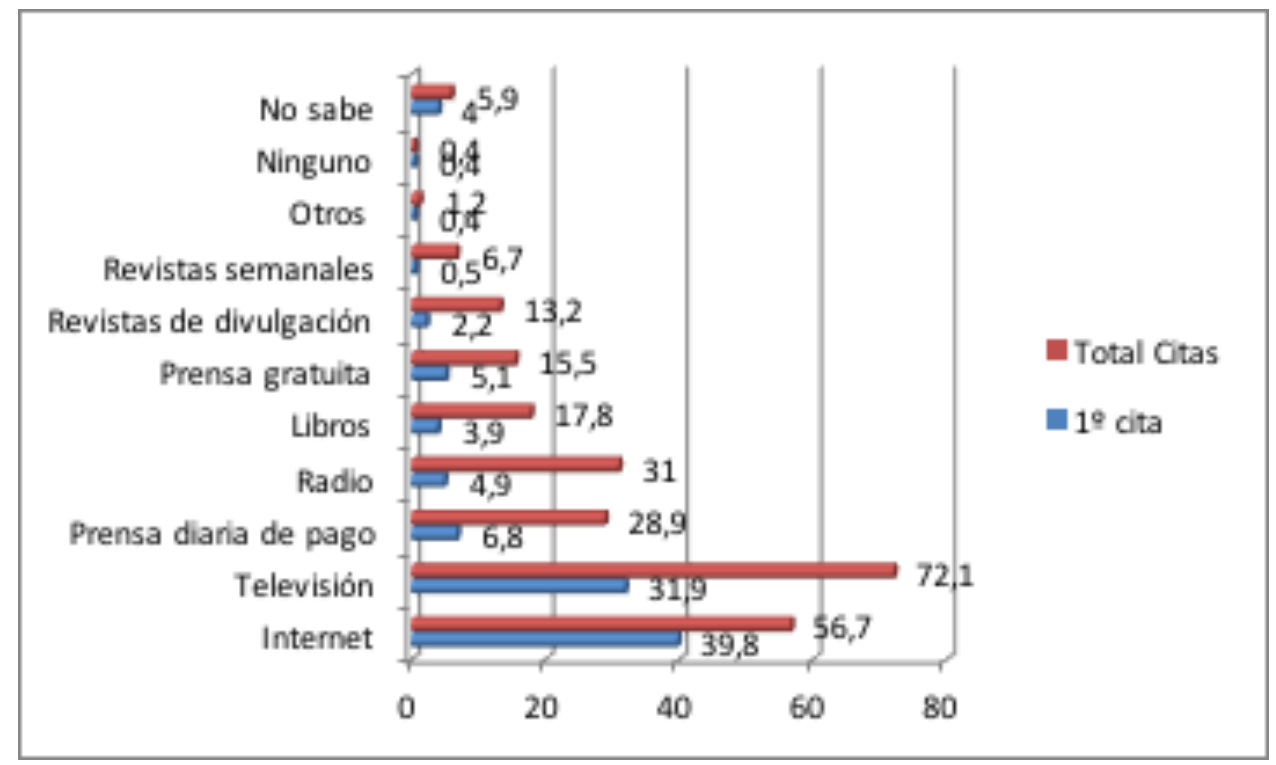

Fuente: FECYT (2015) 
En 2004 la televisión era, con un 39,1\%, el medio que inspiraba a los encuestados más confianza para informarse sobre ciencia y tecnología, seguido de las revistas de divulgación $(22,6 \%)$, Internet $(22,2 \%)$, la radio $(18,2 \%)$ y la prensa (15,8\%). Diez años después dicho ranking ha experimentado un cambio de tendencia: está encabezado, con una puntuación de 1 a 5 , por las revistas de divulgación $(4,20)$, seguidas de la radio $(3,47)$.

\section{Gráfico 4. Nivel de confianza en los medios para informarse sobre ciencia y tecnología}

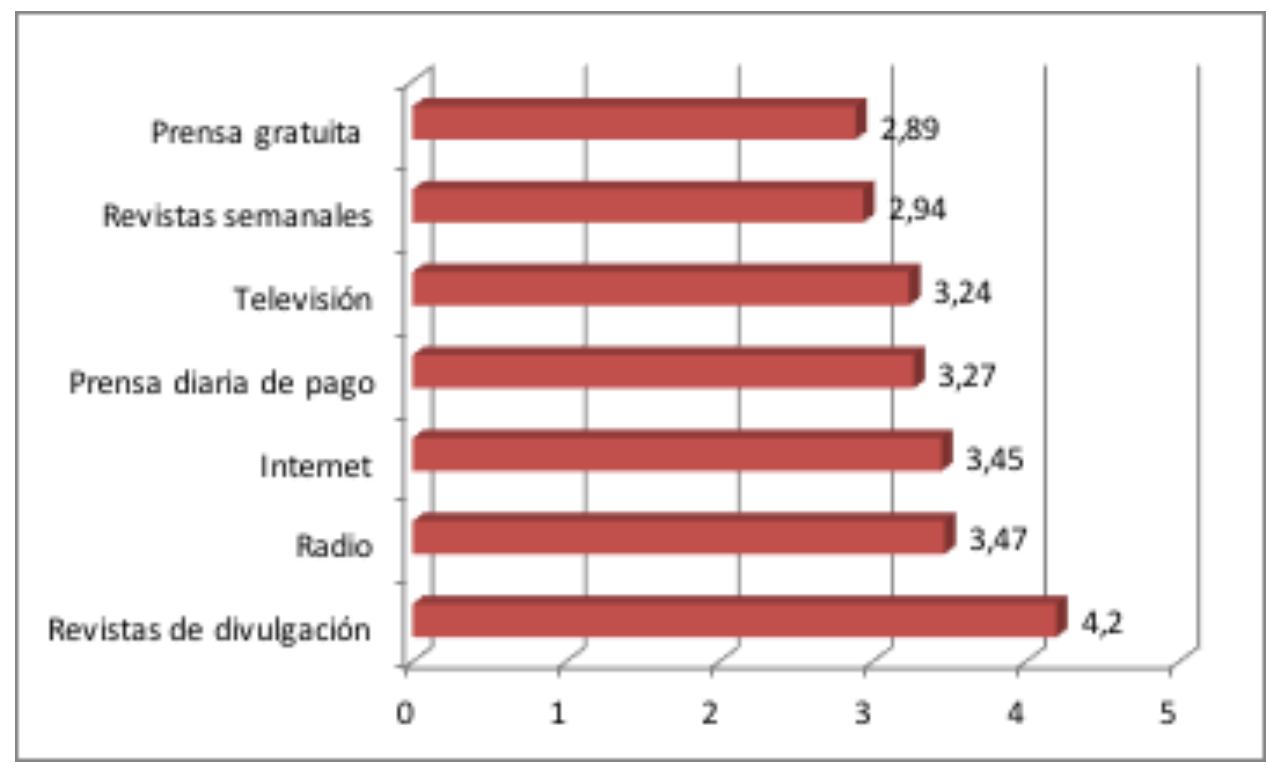

Fuente: FECYT (2015)

En 2004 un 34,1\% de los encuestados consideraban que la radio prestaba una atención insuficiente a la información científica, frente a un $46,5 \%$ que la valoraba como su- ficiente. La percepción de que hay un déficit de información científica en este medio se ha acrecentado una década después, tal y como se observa en el siguiente gráfico.

\section{Gráfico 5. Percepción de la atención que prestan los medios a la información científica}

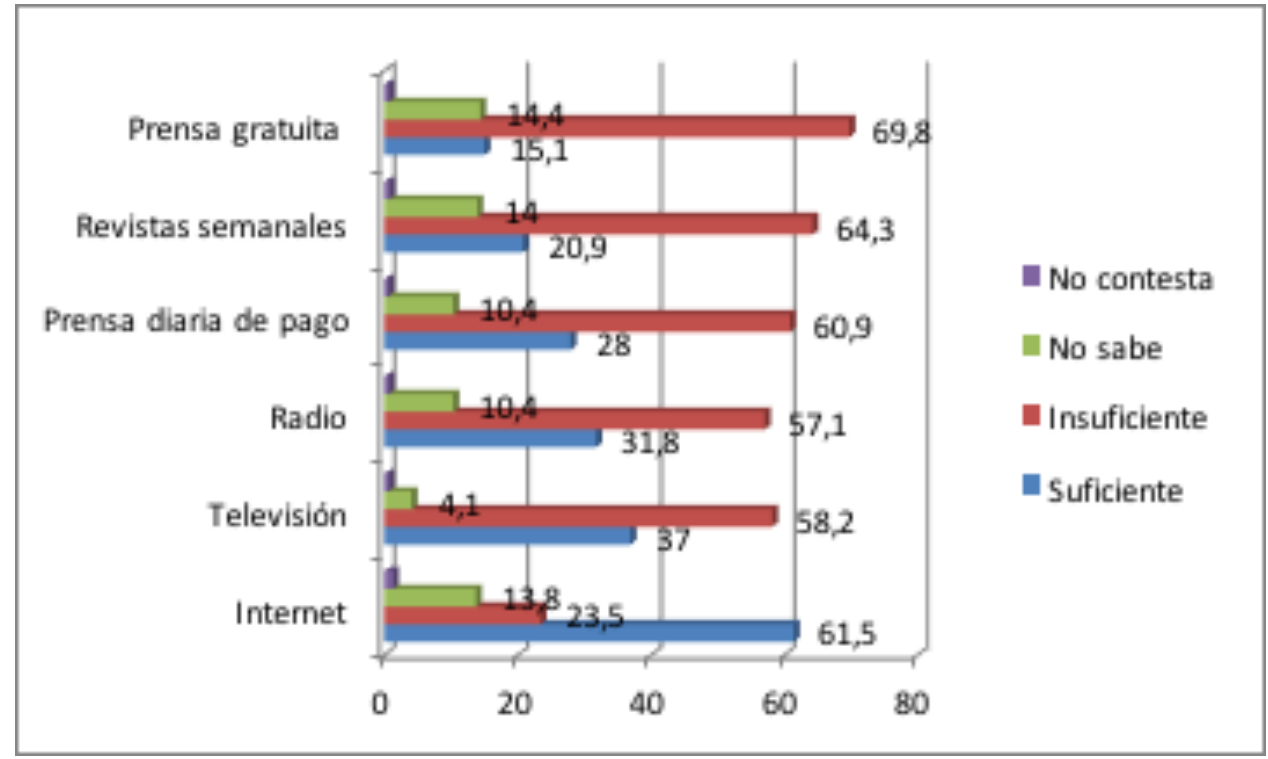

Fuente: FECYT (2015) 
La comunicación científica es una línea de investigación muy desarrollada internacionalmente. Cabe citar, entre otros, los trabajos de Brossard y Scheufele (2013), Guenther y Ruhrmann (2013), Gans (2010) o Bucchi y Mazzolini (2003). Está considerada un área de investigación en constante crecimiento, sobre cuya definición han disertado Burns, O'Connor, y Stocklmayer (2003), y en la que, según Olvera y López (2015), se pueden diferenciar tres grandes aspectos de investigación: la cobertura científica en los medios desde el punto de vista cuantitativo y cualitativos, entre cuyos trabajos citan a Fishman (1980) o a Suleski e Ibaraki (2010); la precisión y el rigor de los medios en la representación de la ciencia, cuestión abordada, entre otros, por Ryan (1979); o la relación entre los científicos y los periodistas (Nisbet et al., 2002).

González, Valderrama y Aleixandre (2007) han analizado la producción científica española sobre divulgación de la ciencia, identificando los principales agentes, canales de difusión y ámbitos abordados. Cabe citar, además, las investigaciones desarrolladas por González, Luján y López (1996), Elías (2008), De Semir (1996) y Calvo (1997b), entre otros. Entre las líneas de investigación que se han desarrollado más recientemente en este país figuran la comunicación de la ciencia 2.0 (López \& Olvera, 2015), las informaciones sobre ciencias de la salud en los medios (Revuelta, 2006) o la situación de determinadas comunidades autónomas (Mariño, 2016).

La alfabetización se ha abordado en la modalidad transmediática (Echazarreta, 2014) y el papel de la radio como instrumento educativo y una herramienta básica para el periodismo y la divulgación científica ha sido objeto de estudio por parte de Díaz (2004). Sin embargo, tras realizar la revisión bibliográfica correspondiente, se está en condiciones de afirmar que no se ha prestado atención, hasta el momento, al papel de la radio pública de ámbito estatal en España como medio de empoderamiento de las personas a través de la alfabetización científica, ni se ha realizado un estudio exploratorio-descriptivo sobre la oferta de su programación especializada en esta temática, ni se han estudiado en profundidad y caracterizando algunos de sus espacios de mayor recorrido.

\section{METODOLOGÍA}

Por ello, el objetivo de este estudio es, en primer lugar, conocer si la radio pública de ámbito estatal en España está apostando por la divulgación científica para sus oyentes y, en segundo lugar, definir cómo lo está haciendo, es decir, a través de qué tipo de espacios, en qué emisoras, con qué periodicidad y en qué franja horaria. Ello permitirá, además de identificar las experiencias que se están desarrollando, reflexionar sobre las fortalezas y debilidades de la radio pública estatal en esta materia y las nuevas posibilidades que podría explorar. Es, por tanto, una investigación académica con un enfoque teórico y profesional (Wimmer y Dominick, 1996).

Se parte de la hipótesis de que, en consonancia con el incremento del interés informativo por la I+D+i y de la concienciación de la comunidad investigadora de la necesidad de dar a conocer su labor, se han incorporado espacios en la radio pública estatal en España dedicados a este tema.

Para analizar si la radio pública de ámbito estatal en España está cumpliendo su servicio público de alfabetización y fomento de la cultura científica se ha seleccionado como objeto de estudio la Corporación de Radio Televisión Española (RTVE), sociedad mercantil estatal que, tal y como se indica en su página web, "tiene encomendada la misión de ofrecer y garantizar el servicio público de radio y televisión de titularidad del Estado. Su capital social es de titularidad íntegramente estatal. Sus grandes áreas de actividad son la televisión, la radio, rtve.es, el Instituto RTVE y la Orquesta Sinfónica y Coro RTVE".

Según Ortiz (2012), ha venido ofreciendo 
ciertos contenidos de proximidad a la ciudadanía. En este contexto, la primera preguntas de investigación que nos formulamos es si entre dichos contenidos se encuentran los científicos.

Para dar respuesta a ello, se realizó una búsqueda pormenorizada a septiembre de 2016 en las parrillas de las seis emisoras de radio de RTVE: Radio Nacional (centrada en contenidos sociales, culturales y deportivos), Radio Clásica (dedicada a la vida cultural española e internacional y la música clásica en particular), Radio 3 (focalizada en la cultura y a la música joven), Ràdio 4 (emite exclusivamente para Cataluña), Radio 5 Todo Noticias (radiofórmula informativa de RNE) y Radio Exterior de España (emite para todo el mundo). El objeto de estudio, por tanto, son emisoras que, siguiendo la clasificación propuesta por Tenorio (2008: 48-54), se clasifican como gubernamentales con una programación generalista.

En segundo lugar, de entre los 46 espacios localizados se hizo una selección en base los siguientes criterios: 1) que la cobertura temática sea la I+D+i en general, 2) singularidades del programa 3) que sea de largo recorrido. Los seleccionados fueron "A hombros de gigantes" y "Entre probetas", que se definieron y caracterizaron a través del uso dos técnicas cualitativas: la entrevista con sus promotores y el análisis de contenido.

La entrevista se realizó en septiembre de 2016 a través de un cuestionario remitido por correo electrónico a Manuel Seara Valero ("A hombros de gigantes") y a José Antonio López ("Entre probetas"). Se formularon preguntas relacionadas con el espacio, entre las que figuraron ¿Cuándo se comenzó a emitir y por iniciativa de quién?, ¿Cuál es su público objetivo y cuál su principal cometido? ¿Cambió el espacio en algún momento de hora y día de emisión?, ¿A qué perspectivas futuras se enfrenta? Además, se les preguntó por la valoración que tienen la cultura científica de la sociedad española, las áreas científicas que consideran más fáciles y más difíciles de comunicar, las ventajas e inconvenientes que aprecian en la radio para divulgar la I+D+i y la percepción del sistema mediático en España en relación con los espacios destinados a la difusión de la ciencia

El análisis de contenido fue de cuatro programas. En el caso de "A hombros de gigantes" los emitidos los días 5, 12, 19 y 26 de septiembre y en el caso de "Entre probetas" los días 7, 14, 21 y 28 de septiembre. Se ha recurrido a esta técnica para alcanzar los siguientes objetivos:

- Determinar, siguiendo la clasificación de Tenorio (2008: 40-43), el tipo de programa, y, siguiendo la clasificación propuesta por Chimeno (1997: 45-46), a qué nivel medios pertenece cada espacio

- Conocer y analizar la estructura del espacio: duración y bloques

- Identificar las principales áreas científicas a las que da cobertura y a través de qué géneros periodísticos. Para ello, se recurrió a la clasificación propuesta por Pousa e Yaguana (2013), que diferencian entre géneros radiofónicos monologales (noticia, crónica, reportaje, informativos de opinión); apelativos o dialogales (la entrevista) y coloquiales mixtos de información y opinión (mesa redonda, debate, tertulia y encuesta)

- Saber a qué tipo de fuentes de información recurren para obtener el contenido del espacio, diferenciando entre exclusivas o compartidas (Túñez, 1999)

- Observar si promueven la participación de los oyentes en el espacio, formulando preguntas o con secciones específicas que fomenten su interactividad

- Aproximarse al uso que hacen de la radio a la carta y de las redes sociales (si las emplean), y si hacen referencia a ello en la emisión del espacio 


\section{RESULTADOS}

La búsqueda en las parrillas de las seis emisoras y en el buscador de programas de radio RTVE bajo la categoría de Ciencia y Tecnología ha dado como resultado la localización de 46 espacios, si bien al indagar en cada uno de ellos se constata que sus temáticas son muy variadas: desde las ciencias de la salud a las tecnologías de la información y la comunicación, pasando por la sexología, el medio ambiente, la neurociencia.

\section{Tabla 1. Espacios emitidos en las emisoras de RTVE bajo la categoría Ciencia y Tecnología (2016)}

\section{Título}

A hombros de gigantes

A su salud

Alimentación saludable

Alimento y salud

Amigos de radio Exterior

Animales y medio ambiente

Blogueros

Buenas hierbas

Cartas de afrodita

Ciencia y acción

Cita previa

Cómete el mundo

Desde cero

Doble hélice

El bosque habitado

El buscador de Radio 5

El laboratorio de JAL

El ojo de la aguja

Entre probetas

España.com en REE

Fallo de sistema

Farmacia Abierta

Fauna cercana

Generación verde

Inquietamente

Investigación y criminología

La ciencia en la alcoba

La felicidad

L'Altra Ràdio

Lógica paleontológica

Mi gramo de locura

Ondas de ayer

Planeta vivo

Por todo lo alto

Red abierta

Red Natura 2000

Reserva Natural

Respuestas de la ciencia

Secretos del cerebro

Seguridad del internaura

Sostenible y renovable

Un laboratorio en mi cocina

Vademécum

Vida verde

3.0

5.0

\section{Emisora}

RNE

Radio 5

Radio 5

Radio 5 y Exterior

Radio Exterior

Radio 5

Radio 5

Radio 5

Radio 5

Radio 5

Radio 5

Radio 5

Radio 5

Radio Exterior

Radio 3 y Radio Exterior

Radio 5

Radio 5

Radio 5

Radio 5 y Radio Exterior

Radio Exterior

Radio 3

Radio 5

Radio 5

Radio 5

Radio 5

Radio 5

Radio 5

Radio 5

Ràdio 4

Radio 5

Radio 5

Radio 5

Radio 5

Radio 5

Radio 5

Radio 5

Radio 5 y Radio Exterior

Radio 5

Radio 5

Radio 5

Radio Exterior y Radio 5

Radio 5

Radio 5

Radio Exterior

Radio 3

Radio 5

Radio

Exterior, RNE

\section{Hora de emisión}

Lunes de 02.00 a 03.00 horas

Lunes, miércoles y viernes a las $17.52,17.55$ y 10.56 horas

Martes a las 11.52 horas

Domingo de 12.35 a 13.00 horas y de 03.30 a 04 horas, respectivamente

Lunes de 02.00 a 03.00 horas

Sábado a las 16.17 horas y domingo a las 10.35 horas

Jueves a las 10.07 horas

Miércoles a las 18.37 horas

Jueves a las 01.35 horas

Viernes a las 18.47 horas

Viernes a las 12.55 horas

Sin hora de emisión fija

Viernes a las 17.47 horas

Viernes de 04.30 a 05.00 horas

Domingo de 11.00 a 12.00 horas y sábado de 05.00 a 06.00

horas, respectivamente

Lunes a jueves a las 11.07 horas

Lunes, miércoles y viernes a las 10.07 horas

Sin hora de emisión fija

Miércoles de 00.05 a 00.30 horas y domingo de 03.00 a 03.30

horas, respectivamente

Lunes a viernes de 07.30 a 08.00 horas

Domingo de 12.00 a 13.00 horas

Lunes a las 17.47 horas

Martes y jueves a las 16.37 horas

Jueves a las 18.52 horas

Martes a las 12.37 horas

Jueves a la 01.45 horas

Miércoles a las 04.27 horas

Domingo a las 10.50 horas

Domingo de 22.00 a 23.00 horas

Viernes a las 18.47 horas

Jueves a las 12.37 y viernes a la 01.52 horas

Martes a las 18.20 horas

Martes a las 17.43 horas

Miércoles a las 10.51 horas

Martes a la 01.05 horas

Domingo a las 09.20 horas

Miércoles de 19.05 a 19.30 horas y jueves de 06.30 a 07.00

horas, respectivamente

Sin hora de emisión fija

Miércoles a las 17.27 horas

Domingo a las 10.47 horas

Domingo de 12.00 a 12.30 horas y martes de 00.30 a 01.00

Domingo a las 13.05 horas

Martes a las 11.37 horas

Jueves de 04.00 a 05.00 horas

Sólo se emite online

Sin hora de emisión fija

Viernes de 00.05 a 00.30 horas, sábado de 11.30 a 12.00 horas domingo a de 13.00 a 13.30 horas, respectivamente 
Tras realizar una primera aproximación a cada espacio, de los 46 se seleccionaron como objeto de estudio los dos que, a nuestro criterio, más se enfocan a la $I+D+i$ en general y tienen un largo recorrido que avala su labor y su éxito: "A hombros de gigantes" $y$ "Entre probetas".

"A hombros de gigantes" comenzó a emitirse el 7 de septiembre de 2007 en Radio 5 y en Radio Exterior. En septiembre de 2012 se trasladó a RNE, donde acaba de iniciar su décima temporada, emitiéndose ininterrumpidamente con una periodicidad semanal de septiembre a julio los lunes de 02.00 a 03.00 horas. No cuenta con ayudas públicas de entidades, sino que se hace dentro de la programación de RNE y a cargo del presupuesto de RTVE.

Se presenta como "un espacio de radio dedicado a la ciencia". Durante el programa hay ráfagas con mensajes como "A hombros de gigantes pone la ciencia al alcance de todos", "A hombros de gigantes no dejará de sorprenderte" o "El espacio de las mentes curiosas".

Su director, productor, realizador y presentador es Manuel Seara Valero, licenciado en Biología y máster en Periodismo Radiofónico. El programa cuenta con una amplia nómina de colaboradores, desde investigadores a filósofos pasando por personal con diferentes perfiles profesionales relacionados con la I+D+i del Consejo Superior de Investigaciones Científicas y del Servicio de Información y Noticias Científicas.

Su cometido es ser un espacio pegado a la actualidad, que cuente en tono divulgativo los últimos avances científicos, dando voz a sus protagonistas. También pretende dar a conocer los centros de investigación que hay en España, el trabajo que llevan a cabo y su repercusión en nuestra vida. Para ello, se dirige al público general interesado por la ciencia. Según palabras de Manuel Seara, "una audiencia muy crítica, en el mejor sentido de la palabra. Apasionada, con una gran curiosidad, y una mente muy despierta". El espacio tiene una duración de cerca de una hora, con una estructura fija: una entrevista, de unos 20 minutos de duración, y un bloque de secciones de los colaboradores, de unos cinco minutos cada uno.

En los cuatro programas objeto de análisis de contenido Manuel Seara Valero entrevistó a María Blasco, directora del Centro Nacional de Investigaciones Oncológicas; Mauricio Antón, paleoartista especializado en la reconstrucción científica de la vida del pasado; José Hernández, ingeniero de operaciones y calibración de Gaia, e Inés Rodríguez Hidalgo, directora del Museo de la Ciencia de Valladolid. Las entrevistas versaron sobre sus líneas de investigación o aspectos de su trabajo de actualidad.

En las secciones con colaboradores $\mathrm{Ma}-$ nuel Seara Valero les formula preguntas, estableciéndose un diálogo entre ambos. En algunos casos el tema de la sección está relacionado con la actualidad científica, por ejemplo, hallazgos recientes, publicaciones en revistas o celebraciones de congresos. En los cuatro programas objeto de análisis de contenido participaron las siguientes personas con las siguientes temáticas:

- José Antonio López Guerrero, profesor de la Universidad Autónoma de Madrid y director de Cultura Científica del Centro de Biología Molecular Severo Ochoa. Explicó en tono divulgativo cuestiones relacionadas con los citomegalovirus, la posibilidad de que el alzheimer sea una enfermedad transmisible, el virus de Crimea-Congo, causante la muerte de un excursionista en Ávila picado por una garrapata, y los detalles de un artículo publicado en Nature que trata de regulación y reglamentación en la investigación y tratamientos con células madre.

- Jesús Zamora, catedrático de Filosofía de la Ciencia en la Universidad Nacional de Educación a Distancia, reflexionó sobre la llamada "voluntad general", su concepto y plasmación.

- Pedro Gargantilla, profesor de la Univer- 
sidad Europea de Madrid y médico del Hospital del Escorial, abordó la relación de Cervantes con la medicina y cómo se vio plasmada en "El Quijote".

- Sección "Geología". Jesús Martínez Frías, investigador del Instituto de Geociencias, explicó en tono divulgativo diversas cuestiones científicas sobre la luna y sus recursos y sobre los geoparques y su importancia para la conservación y el desarrollo.

- Bernardo Herradón, investigador en el Instituto de Química Orgánica, habló sobre el libro "El sistema periódico", de Primo Levy.

- Javier Ablanque, de la Universidad Politécnica de Madrid, habló de Clarence Dally, un ayudante de Edison que fue expuesto a tantos experimentos de rayos $X$ que acabaron provocando la amputación de uno de sus brazos y la muerte por cáncer.

En la sección "El Origen y la Evolución de la Vida" Carlos Briones, investigador del Centro de Astrobiología, explicó qué son las células eucariotas y las mitocondrias, mientras en "Historia de la Ciencia" Nuria Martínez Medina, diplomada en Magisterio y licenciada en Historia del Arte, presentó las figuras y trayectorias científicas de Karl Wilhelm, biólogo y descubridor de los cromosomas; Laurent, descubridor del antraceno; Albert Von Kölliker, pionero en los estudios de citología e histología; Hermann Kopp, considerado el primer gran historiador de la Química, y Sainte-Claire Deville, quien diseñó hornos de alta temperatura en los que estudió las propiedades de numerosas sustancias.

En representación del Servicio de Información y Noticias Científicas intervinieron Adelina Marcos, quien informó sobre cómo los perros también necesitan no ser agobiados por los niños, y Eva Rodríguez, quien expuso el curioso comportamiento del oso cavernario.

En la sección "Revista de prensa" los res- ponsables de "Investigación y Ciencia" y "Quo", Ernesto Lozano y Jorge Alcalde, avanzaron los contenidos de las revistas para el mes de octubre; en "Moléculas imprescindibles para la vida" Álvaro Martínez del Pozo, de la Universidad Complutense de Madrid, disertó sobre la popular vitamina C, y en "Mujeres y Ciencia" Eulalia Pérez Sedeño, investigadora del Instituto de Filosofía, trazó el perfil de Ynes Mexia, conocida por sus expediciones por todo el continente americano.

Por tanto, la información recogida a través del análisis de contenido ha revelado, en primer lugar, la participación de una amplia representación de personal vinculado a diferentes agentes del sistema de $\mathrm{I}+\mathrm{D}+\mathrm{i}$ de España con una inquietud y apuesta por la divulgación científica, cuya intervención está en tono momento moderada e incentivada por el presentador, Manuel Seara Valero. Esto dota al programa de un carácter interinstititucional, multiinstitucional, multidisciplinar y transdisciplinar.

Se abordan todos los temas científicos, sin exclusión, presentado atención tanto a las Humanidades y Ciencias Sociales como a la Biología, pasando por la Ciencia y Tecnología Físicas, entre otras. También, se da visibilidad tanto a personal investigador tanto de la actualidad como del pasado. Se pretende dar a conocer y poner en valor, por tanto, todas las áreas y todos los perfiles de hombres y mujeres dedicados a la I+D+i a lo largo del tiempo.

Para ello, el espacio obtiene información de fuentes muy variadas: noticias de agencia, notas de prensa, revistas científicas, ruedas de prensa, informaciones particulares...Se opta, así, por una mezcla de fuentes exclusivas y compartidas, y la información se difunde recurriendo a géneros radiofónicos monologales y apelativos o dialogales, teniendo la entrevista un peso clave, con casi el $50 \%$ de la duración del programa.

El oyente de "A hombros de gigantes" tiene 
la posibilidad de escuchar el espacio tras su emisión a través de http://www.rtve.es/alacarta/, donde se dispone de un buscador de programas desde el 22 de abril de 2008. En total, hay 566 programas disponibles para el usuario a través del servicio de radio a la carta online de RTVE.

En cuanto al oyente, el presentador hace mención durante el programa a que "pueden enviar sus sugerencias a través del email", pero en los cuatro programas objeto de estudio no se ha identificado ninguna iniciativa para fomentar su participación. Esta, sin embargo, sí se ha empezado a canalizar desde 2013 a través de las redes sociales, donde el programa tiene su propia página en Facebook, "Programa A hombros de gigantes". A 30 de septiembre contaba con 1.342 seguidores. Desde este espacio se le ofrece a los usuarios una síntesis y un enlace al podcast de cada programa y se comparten las últimas noticias y eventos científicos.

Los programas objeto de estudio generaron la siguiente actividad por parte de los seguidores del espacio en esta red social: el post publicado acerca del programa del 4 de septiembre tuvo 37 Me Gusta, 20 Comentarios y fue tres veces compartido; el del 12 de septiembre generó 14 Me Gusta, 3 comentarios y fue compartido dos veces; el del 19 de septiembre 7 Me Gusta y dos veces compartido, y el del 25 de septiembre 18 Me Gusta, 6 Comentarios y una vez compartido. En general, los comentarios hacen referencia a cuestiones tratadas en el programa y a consultas sobre los invitados. Además, dado que coincide con el inicio de la décima temporada del mismo, también son de felicitación.

En cuanto a la evolución de "A hombros de gigantes" durante sus diez años de emisión, Manuel Seara Valero apunta que "ha ido ligada, fundamentalmente, a la incorporación de colaboradores, con sus respectivas secciones, y a su difusión en la Red a través de los podcats". Sus objetivos, avanza, pa- san por "incrementar el presupuesto para aumentar las colaboraciones y ubicarse en una franja horaria que permita alcanzar a una mayor audiencia".

El principal inconveniente de la radio para divulgar la ciencia considera que "es el carecer de imágenes o textos en los que apoyarse" y sus puntos fuertes cree que "radican en ser un medio natural y muy personal, que tiende a la afectividad o la persuasión; barato, al alcance de la mayoría; inmediato y directo en su difusión". Destaca también que "permite la interacción con el oyente y no le interfiere en sus tareas cotidianas mientras escucha".

En relación al nivel de cultura científica de la sociedad en España, recalca que "es muy bajo y los medios de comunicación podemos contribuir a mejorar esa percepción".

"Entre probetas". Se inició en 2006 como un microespacio de tres minutos diarios que continúa en la actualidad, bajo el nombre "El LAB de JAL". En septiembre de 2014 comenzó a emitirse en su formato actual, con 25 minutos de duración, los miércoles a las 00.05 horas en Radio5 y los domingos de 03.00 a 03.30 horas en Radio Exterior. No cuenta con el patrocinio de ninguna entidad.

Se trata de una iniciativa personal de José Antonio López Guerrero, profesor e investigador del Departamento de Biología Molecular de la Universidad Autónoma de Madrid y director de Cultura Científica del Centro de Biología Molecular Severo Ochoa. Colabora, como se ha expuesto anteriormente, en "A hombros de gigantes". Él es el director, productor, realizador de "Entre probetas". Colaboran en el espacio Ana de las Heras, bióloga y periodista, y Elena Campos, investigadora posdoctoral del Centro de Biología Molecular Severo Ochoa.

Su cometido es contribuir a romper el mito de que la ciencia y la tecnología son aburridas y difíciles de comprender, así como consolidar la divulgación científica desde las emisoras públicas. Para ello, el progra- 
ma se dedica a divulgar e informar de los principales logros e hitos científicos, recurriendo para ello al humor y a la diversión. Al final de cada espacio el presentador y las colaboradoras dicen: "Contra los charlatanes y pseudociencia, leal ciencia. Escuchen ciencia y viva ciencia".

"Entre probetas" se dirige, según palabras de su promotor "al público general, no necesariamente cultivado en los temas propuestos, que tenga interés en incrementar su cultura".

El espacio, a lo largo de sus 25 minutos, consta de entrevistas y conversaciones entre el presentador y las colaboradoras acerca de temas de actualidad.

En los cuatro programas objeto de análisis de contenido José Antonio López Guerrero entrevistó a Juan Antonio Aguilera, profesor de Bioquímica y Biología Molecular de la Universidad de Granada; a Carlos Dotti, investigador del Centro de Biología Molecular Severo Ochoa y a Ion Arocena, director general de Asebio, una de las entidades organizadoras de Biospain 2016.

Se abordaron las siguientes temáticas, a través de conversaciones entre el presentador y las colaboradoras: la relación de la ciencia y el cine, los cometidos de Sociedad Española de Reumatología, la celebración el Congreso Mundial sobre Virología, los primeros casos del virus de Crimea-Congo, el evento la Noche de los Investigadores y el reciente acuerdo de transparencia sobre el uso de animales en experimentación científica presentado por Cotec.

Por tanto, la información recogida ha revelado que el espacio presta atención a diferentes áreas científicas a través, sobre todo, de conversaciones informales entre el presentador y las colaboradoras. Según su presentador, "damos difusión a cualquier tema que se pueda emitir con base científica, prestando especial atención a visualizar enferme- dades raras y a luchar contra la pseudociencia".

Las fuentes de las que obtiene información son tanto exclusivas como compartidas, y los contenidos se difunden recurriendo a géneros radiofónicos monologales y apelativos o dialogales.

El oyente de "Entre probetas" tiene la posibilidad de escuchar el espacio tras su emisión a través de http://www.rtve.es/alacarta/, donde se dispone de un buscador de programas desde el 5 de agosto de 2008. En total, 891 programas disponibles para el usuario a través del servicio de radio a la carta online de RTVE.

Durante el espacio se dice que el oyente "puede dejar sus propuestas o hacernos sugerencias en nuestro correo electrónico", pero no se han identificado en los cuatro programas objeto de estudio iniciativas para fomentar su participación. Esta, sin embargo, sí se ha empezado a canalizar desde febrero de 2016 a través de las redes sociales, donde el programa tiene su cuenta en Twitter @EntreProbetas. A 30 de septiembre de 2016 contaba con 152 seguidores y se habían publicado 17 tweets, sobre todo, para avanzar contenidos de los programas. La interacción ha sido escasa.

Su presentador considera que las principales ventajas del medio radiofónico para difundir la ciencia son "su lenguaje directo, inmediato, casi más personal, flexible" y como inconvenientes apunta "la competencia con emisoras y programas con mayor aceptación".

En cuanto a la situación del sistema mediático en España en relación con los espacios en los diferentes soportes destinados a la difusión de la ciencia, cree que son "deficientes o imprecisos. Se valora poco y las emisiones de estos programas suelen ser en franjas horarias intempestivas". 


\section{DISCUSIÓN}

Un estudio de la Comisión Europea encargado a un grupo de expertos en 2006 proponía que la alfabetización mediática reconociera tres pilares fundamentales: la educación en medios como fuentes de instrucción para la adquisición de nuevas capacidades, la potenciación de las capacidades de creación y producción mediática y la participación y actividad en los medios y en la sociedad como elemento clave de la ciudadanía activa. Tal y como se ha revelado en este estudio, el primero de esos principio se está cumpliendo a través de la radio pública de titularidad estatal en España en el ámbito de la alfabetización científica. El tercero es, probablemente, el futuro de la ciencia en la radio.

La sociedad del siglo XXI ni puede ni debe permanecer de espaldas a los avances científicos y tecnológicos que se están produciendo. Tiene que conocerlos y comprenderlos en la medida de las posibilidades que le brinde su formación. Pero no sólo debe estar al tanto de los grandes hitos científicos, tales como la confirmación de la teoría gravitacional de Einstein (febrero de 2016) o el descubrimiento del bosón de Higgs (marzo 2013). Cada día, la ciencia es noticia tanto a través de hechos que se producen en el ámbito local como de otros que lo hacen en el internacional. Una buena recolección de esos hechos es la que se publica en el Anuario de la Agenca SINC. La ciencia es noticia (FECYT, 2015).

Acercar la ciencia a la sociedad no es una tarea fácil, más aún a la vista de datos como los de la última encuesta de Percepción Social de la Ciencia y la Tecnología de la FECYT, donde se apunta que el 35,9\% de los encuestados declara que están poco o nada interesados por la ciencia porque no la entienden y donde se pone de manifiesto que la sociedad española todavía no sabe si son verdaderas o faltas afirmaciones como "los seres humanos provienen de especies animales anteriores" o "el sol gira alrededor de la tierra" (FECYT, 2015: 317-362).
Hace dos décadas, Calvo (1997b) ya vertía de un retraso de la divulgación de la ciencia en relación con los avances científicos actuales, a la par que hablaba de un desfase entre la sociedad y la comunidad científica. Así continúa siendo, tal y como queda patente por estos datos. Frente a esta situación, es importante llevar la ciencia al público.

Hay, por tanto, un largo camino por avanzar, primero, en la alfabetización mediática básica de la sociedad y, después, en el mantenimiento de una cultura científica acorde a la formación en otras áreas de la ciudadanía contemporánea. Los medios, sobre todo los de titularidad pública, tienen un cometido que cumplir, actuando con proactividad y empleando el tiempo y los recursos de los que disponen en comunicar la I+D+i.

Desde sus inicios, la radio ha sido un ideal instrumento educativo, y como tal ha sido utilizado por intelectuales y comunicadores de la ciencia (Díaz, 2004). Ahora, en un panorama mediático altamente competitivo con nuevos hábitos de consumo de medios, la radio continúa siendo un instrumento educativo que puede, además, aprovecharse de las nuevas posibilidades tecnológicas para atender más y mejor las necesidades de su audiencia.

Alboukrek (1991) atribuye entre los objetivos de la divulgación científica el de crear una atmósfera de estímulo a la curiosidad por la ciencia y abrir caminos a la participación del desarrollo cultural universal, cometidos a los que Calvo (1997a) añade establecer una conciencia científica colectiva, cohesionar a los grupos sociales o ser un factor del desarrollo cultural. Los programas "A hombros de gigantes" y "Entre probetas" van en esa línea.

No obstante, no lo tienen nada fácil. La multiplicación de la información y el entorno multipantalla pueden estar creando una saturación para las mentes y los sentidos, y estar conduciendo en ocasiones a una es- 
pecie de embotamiento perceptivo que, lejos de ayudar al aprendizaje, es una barrera para su conocimiento (Pérez Tornero, 2008: 17). La radio, además, presenta el añadido de ser un medio con un mensaje fugaz, que no se visualiza y que se consume, frecuentemente, mientras se realiza otras actividades.

Hace dos décadas Martínez (1997) consideraba que la radio aún no había sido capaz de ofrecer una mayor alternativa de programas y Calvo (1997a:170) apuntaba que en la programación radiofónica podían y debían figurar espacios de divulgación científica en sus respectivas formas: divulgación pura de conocimientos científicos; mensaje científico a la sociedad; información de actualidad científica y programas de opinión y crítica científica. Los programas "A hombros de gigantes" y "Entre probetas" optan, sobre todo, por la segunda y tercera forma.

Estos programas tienen el siguiente perfil de oyente, en consonancia con el de la radio española (AIMC, 2016): el 51,8\% son hombres y el $48,2 \%$ mujeres, siendo mayoritarios los oyentes entre 35 y 44 años (22,2\%). Según este estudio, el ranking de emisoras de radio generalista en España está liderado por la Cadena SER, seguida de la Cadena COPE, Onda Cero y RNE. Por tanto, uno de los programas objeto de estudio, "A hombros de gigantes" se emite en la cuarta emisora líder en España.

El sistema español de I+D+i y los medios de comunicación tienen la responsabilidad de mejorar el proceso de comunicación social de la ciencia para alfabetizar y atender las necesidades formativas del público no especializado. Esta responsabilidad compete a ambos: el sistema debe mostrar una actitud receptiva hacia la difusión divulgativa de su labor y los medios deben crear espacios especializados para ello, algo incuestionable, sobre todo, en los medios públicos. Ambos, el sistema de I+D+i y la radio pública, se sostienen con fondos públicos. Por tanto, les toca, más que a otros, rendir cuentas de en qué se emplea el dinero de los contribuyentes y contribuir a hacer su día a día más culto desde el punto de vista científico, haciendo un uso pedagógico del medio radiofónico con programas de calidad científica.

Este estudio deja constancia de que sí se está presentando atención, en mayor o menor medida y con apuestas más o menos claras, a la divulgación científica desde la radio pública estatal en España. Ahora bien, convendría seguir su evolución y abrir nuevas líneas de investigación acerca de lo que sucede en las emisoras privadas. A grandes rasgos, se podría adelantar que no hay una apuesta clara y definida.

Cada medio tiene un contrato con su público, al que intenta satisfacer, proporcionándole lo que se supone que se espera de él cuando lo compra o sintoniza. De alguna forma, los medios se convierten en representantes de sus audiencias (Fundación Cotec, 2006), mientras que la programación es el escaparate de una cadena radiofónica, que apuesta por una nueva radio en la que se rediseñe una programación especializada y basada en nuevos formatos (Peñafiel, 2007: 31). En esa programación especializada tiene cabida, naturalmente, la ciencia, y en esos nuevos formatos los programas entre el género informativo y el magazine para divulgarla.

Las características intrínsecas de la radio lo convierten en un medio que está llamado a desempeñar un papel clave en la alfabetización científica de la sociedad del siglo XXI. Entre las dificultades a las que tendrá que hacer frente figura la fuerte competencia de otros medios. Tal y como dice Pérez Tornero (2008: 18) "la alfabetización mediática exigida por las nuevas circunstancias sólo puede ser fruto de la convergencia de esfuerzos realizada por las instituciones familiares y educativas, los gobiernos y las autoridades que tienen relación con los medios, las asociaciones cívicas, la industria y los profesionales, así como los medios de comunicación". Entre esos medios de comunicación ya está la radio pública de ámbito estatal en España. 


\section{CONCLUSIONES}

"A hombros de gigantes" y "Entre probetas" son ejemplos de los primeros pasos hacia una radio pública estatal en los que se comunica ciencia a través de las ondas. Siguiendo la clasificación de Chimeno (1997), se ha observado en este estudio que son medios de primer nivel o de información general, los que tienen la máxima divulgación y los que recurren a los mínimos tecnicismos.

Son dos apuestas de comunicación científica en radio que coinciden en ser iniciativas de personas con formación científica e inquietud por la divulgación con un perfil polivalente, pues se encargan de todas las labores requeridas para el espacio. En ambos casos, no obstante, cuentan con colaboradores (sobre todo, en "A hombros de gigantes"), lo que sin duda redunda en la calidad del programa al enriquecerlo con diferentes perspectivas.

También, en los dos casos, se afronta el reto de comunicar todas las parcelas de la ciencia: desde las más proclives a la divulgación a las menos, y de dar cuenta de lo que se está haciendo en las diferentes áreas científicas, no sólo en las más populares. Además, los dos se nutren de una mezcla de fuentes compartidas y exclusivas y coinciden en general en apostar por el género de la entrevista.

Otra coincidencia es que los dos espacios se ubican en aquellas franjas que no están consideradas ni de prime time ni de mayores cuotas de audiencia: los días laborales de madrugada.

No obstante, el hecho de que los dos sean ya de largo recorrido, una década, que uno de ellos ("A hombros de gigantes") haya pasado a emitirse en RNE y que otro ("Entre probetas") evolucionase de un microespacio a un espacio de media hora, abre la esperanza a que la programación científica en radio consiga, a base de perseverancia y esfuerzo, ir ganando el espacio que le corresponde en la parrilla.
Ahora falta por ver si estas apuestas de la radio pública se ven acompañadas de otras y si la radio privada se une con firmeza a la labor mediática de divulgar la ciencia. En ello también repercutirá que estos espacios, sin duda innovadores y propios de nuevos tiempos, sean los abanderados en la apuesta por Internet, por las redes sociales y, también, por la interactividad de los oyentes, elementos clave para el futuro de los medios. Que los dos se emitan ya a través de Internet y que empleen alguna de las redes sociales a su disposición permite pronosticar que así será y que la radio del futuro será, también, una radio científica.

\section{REFERENCIAS BIBLIOGRÁFICAS}

- Alboukrek, A. (1991). En la Ciencia. México: Centro Universitario de Comunicación de la Ciencia.

- Bell, D. (1973). The coming of post-industrial society. New York: Basic Books.

- Bellón Rodríguez, A. (2015). El CSIC en Galicia: un ejemplo de comunicación institucional de la I+D+i. Investigación. Revista Investigación. Cultura, ciencia y tecnología, 14, 57-62.

- Berganza Conde, R. (2005). Periodismo especializado. Madrid: Ediciones Internacionales Universitarias.

- Bik HM, Goldstein MC. (2013). An introduction to social media for scientistis. PLOS Biology, 11 (4). http://dx.doi. org/10.1371/journal.pbio.1001535

- Broussard, D. \& Scheufele, DA. (2013). Science, New Media and the Public. Science, 339, 40-45. doi: 10.1126/science.1232329

- Bucchi, M. \& Mazzolini, R. (2003). Big science, little news: science coverage in the Italian daily press, 1946-1997. Public Undersitanding of Science, 12, 7-24.

- Burns, TW, O'Connor, D.J. \& Stocklmayer, S.M. (2013). Science communication: a contemporary definition, Public Understanding of Science, 12, 183-202

- Calvo Hernando, M. (1997a). Manual de Periodismo Científico. Barcelona: Bosch. - Calvo Hernando, M, (1997b). Objetivos 
de la divulgación de la ciencia, Chasqui, 60, 38-40.

- Chimeno, S. (1997). Las fuentes en el proceso de información científica especializada. En VV.AA.: Estudios sobre Información Periodística Especializada. Valencia. Fundación Universitaria San Pablo CEU.

- Díaz, EJ. (2004). La radio y el multimedia, dos alternativas para la divulgación científica, Quark Ciencia, medicina, comunicación y cultura, 34, 40-49.

- De Semir, Vladimir. (1996). '¿Qué hechos merecen ser noticia?' The Lancet [ed. esp.] 29, 185-189.

- Echazarreta Soler, C. (2014). Editorial. Alfabetización transmediática, Communication Papers, 4.

- European Commission (2012). Monitoring policity and research activities on science in Europe. Final synthesis report. Disponible en https://ec.europa. $\mathrm{eu} / \mathrm{research} / \mathrm{science-society/docu-}$ ment_library/pdf_06/monitoring-policy-research-activities-on-sis_en.pdf

- Elías, C. (2008). Fundamentos de periodismo científico y divulgación mediática. Madrid: Alianza Editorial.

- Fernández del Moral, J. \& Esteve Ramírez, F. (1993). Fundamentos de información periodística especializada. Madrid: Editorial Síntesis.

- Falk JH \& Dierking ID. (2010). The 95 per cent solution. American Scientist. Disponible en http://www.americanscientist. org/issues/feature/2010/6/the-95-percent-solution

- Fishman, M. (1980). Manuctaring the News. Estados Unidos: University of Texas Press.

- Fundación Cotec para la Innovación Tecnológica (2006). Comunicar la ciencia. Colección Innovación Práctica. Madrid: COTEC.

- Fundación Española para la Ciencia y la Tecnología (2015). Percepción Social de la Ciencia y la Tecnología 2014. Madrid: FECYT.

- Fundación Española para la Ciencia y la Tecnología (2015). SINC: La ciencia es noticia. Madrid: FECYT.

- Gans, H. (2010). News and the news media in the digital age: Implications for democracy. Daedalus 139 (2), 8-17. DOI: 10.1162/daed.2010.139.2.8.

González García, Ml; Luján López, JL \&López Cerezo, JA. (1996). Ciencia, tecnología y sociedad: una introducción al estudio social de la ciencia y la tecnología. Madrid: Tecnos.

- González Alcaide, G; Valderrama Zurián, JC \& Aleixandre Benavent, R. (2007). La investigación sobre la divulgación de la ciencia en España: Situación actual y retos para el futuro, Arbor, 2007, 738, 861869.

- Guenther, L \& Ruhrmann, G. (2013). Science journalists' selection criteria and depiction of nanotechnology in German media, Journal of Science Communication, 12 (3), 1-17.

- López Pérez, L. \& Olvera Lobo, MD (2015). Comunicación de la ciencia 2.0 en España: El papel de los centros públicos de investigación y de los medios digitales, Revista Mediterránea de Comunicación, 6(2), 165-179. DOI: 10.14198/ MEDCOM2015.6.2.08

Mateo, JL. (2006). Sociedad del Conocimiento, Arbor, 718, 145-151.

- Martínez Costa, MP. (1997). La radio en la era digital. Madrid: El País-Aguilar.

- Mariño Alfonso, X. (2016). (Coordinador). A divulgación da ciencia en Galicia. Santiago de Compostela: Consello da Cultura Galega. doi:10.17075/isdcg.2016.

- Mccombs, M. (2006): Estableciendo la agenda: el impacto de los medios en la opinión pública y el conocimiento. Barcelona: Paidós.

- Méndez, E. (2007). La ciencia como noticia: estrategias discursivas y textuales. La clonación terapéutica. En Delgado Cobos, I. \& Puigvert Pcal, A. (EDS). Ex admiratione et atmicia: homenaje a Ramón Santiago. Madrid: Ediciones el Orto. - Muñoz Torres, JR, (1997). Aproximación al concepto de información periodística especializada. En Esteve Ramírez, F. (COORD), Estudios sobre Información 
periodística especializada. Valencia: Fundación Universitaria San Pablo CEU.

- Nelkin, D. (1990). La ciencia en el escaparate. Madrid: Fundesco.

- Nisbet, M. C., Scheufele, D. A., Shanahan, J., Moy, P., Brossard, D.\& Lewenstein, B. V. (2002). Knowledge, Reservations, or Promise? A Media Effect Model for Public Perceptions of Science and Technology.' Communication Research 29, 584608. DOI: 10.1177/009365002236196.

- Ortiz, MA \& Pérez Ornia JR. (Editores) (2006). Claves para elaborar la información en la radio y la televisión. Madrid: Instituto Oficial de Radio y Televisión. Servicio de Publicaciones de RNE.

- Ortiz Sobrino, MA. (2012): Evolución del modelo de financiación de Radio 5. Del estatuto de radio y televisión de 1980 a la Ley general de comunicación audiovisual de 2010, Icono 14, 3, 383-402.

- Olvera Lobo, MD \& López Pérez, L. (2015): Science journalism: the standardisation of information from the press to the internet. Journal of Science Communication, 14 (3), 1-12.

- Peñafiel Saiz, C. (ED) (2007). Transformaciones de la radio y la televisión en Europa. Bilbao: Servicio Editorial de la Universidad del País Vasco.

- Pérez Tornero, JM. (2009): El nuevo horizonte europeo de la alfabetización mediática, Telos, 79.

- Pérez Tornero, José Manuel (2008): La sociedad multipantallas: retos para la alfabetización mediática, Comunicar, número 31, pp. 15-25.

- Pousa XR \& Yaguana HA. (2013). La radio, un medio en evolución. Salamanca: Comunicación Social Ediciones y Publicaciones.

- Quesada, M. (1998). Periodismo especializado. Madrid: Ediciones Internacionales Universitarias.

- Ramos, C. (1995): Los medios de comunicación: agentes constructores de lo real, Comunicar, 5, 108-112.

- Revuelta, G. (2006). Salud y medios de comunicación en Españ.' Gaceta Sanitaria 20 (1), 203-208.
- Ribas, C. (2002): El periodismo científico y su relación con el proceso de producción de las noticias en los medios de comunicación de masas, Mediatika: cuadernos de medios de comunicación, 8, 499-522.

- Ryan, M. (1979). Attitudes of scientists and journalists towards media coverage of science news, Journalism Quarterly, $56,18-26$.

- Sanmartín, JM. (2003). Periodismo Especializado, el nexo entre conocimiento y sociedad. En De Ramón, M. 10 lecciones de Periodismo Especializado. Madrid: Fragua.

- Solís Garza, H. (2002). Algunas consideraciones sobre lo que solemos llamar ciencias "duras" y ciencias "blandas". Cuadernos de Psicoanálisis.

- Suleski, J \& Ibaraki, M. (2010). Scientists are talking, but mostly to each other: a quantitative analysis of research represented in mass media, Public Understanding Science 19 (1), 115-205. DOI: 10.1177/0963662508096776.

- Tenorio, I. (2008). La nueva radio. Manual completo del radiofonista moderno. Barcelona: MARCOMBO

- Tuñón, A. (1993). L'especialització en periodisme: un canvi de paradigma. Anàlisi, 15, 85-98.

- Túñez, M. (1999). Producir noticias: cómo se fabrica la realidad periodística. Santiago de Compostela: Tórculo Edicións.

- UNESCO (2005). Informe Mundial de la UNESCO "Hacia las Sociedades del Conocimiento", Ediciones UNESCO, París.

- Wimmer, R. \& Dominick J. (1996). La investigación científica de los medios de comunicación. Una introducción a sus métodos. Barcelona: Bosch Comunicación.

\section{Otras referencias}

- RAE http://www.rae.es/

- Declaración de los Derechos Humanos http://www.un.org/es/documents/ udhr/

- Directiva Europea de Servicios Audiovisuales http://eur-lex.europa.eu/ 
- Constitución Española http://noticias. juridicas.com/base_datos/Admin/ constitucion.html

- Ley de la Ciencia https://www.boe.es/ buscar/act.php?id=BOE-A-2011-9617 RTVE http://www.rtve.es/

\section{Agradecimientos:}

Manuel Seara Valero ("A hombros de gigantes") y José Antonio López ("Entre probetas") 